\title{
Genome-wide analysis of the NF-Y gene family in peach (Prunus persica L.)
}

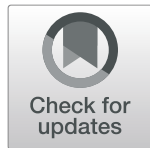

\author{
Miao Li, Guixiang Li, Wei Liu, Xiaomin Dong and Anning Zhang ${ }^{*}$
}

\begin{abstract}
Background: Nuclear Factor $Y(N F-Y)$ is a heterotrimeric complex composed of three unique subunits: NF-YA, NFYB, and NF-YC. The NF-Y transcription factor complex binds to the CCAAT box of eukaryotic promoters, playing a vital role in various biological processes in plants. However, the NF-Y gene family has not yet been reported from the peach genome. The current study identified and classified candidate peach NF-Y genes for further functional analysis of this family.

Results: The current study identified 24 Nuclear Factor Y (NF-Y) transcription factor subunits (6 NF-YA, 12 NF-YB, and 6 NF-YC subunits) in peach. These NF-Y subunits were described with respect to basic physicochemical characteristics, chromosome locations, gene structures, and conserved domains. Based on an analysis of the phylogenetic relationships among peach NF-YS, six pairs of paralogous NF-YS were detected. The expansion of the peach NF-Y family occurred by segmental and tandem duplication. Phylogenetic gene synteny of NF-Y proteins was observed between peach and Arabidopsis, and five pairs of paralogous NF-Y proteins from peach and Arabidopsis were identified. Twenty-four peach NF-YS displayed a diversity of tissue expression patterns. In addition, droughtresponsive cis-elements were observed in peach NF-Y promoters, and 9 peach NF-Y genes were shown to distinctly increase their transcript abundances under drought stress.

Conclusions: This study identified 24 NF-Y genes in the peach genome and analysed their properties at different levels, providing a foundation for researchers to understand this gene family in peach. The up-regulation of 9 NF-Y genes under drought stress indicates that they can serve as candidate functional genes to further study drought resistance in peach.
\end{abstract}

Keywords: Peach, Nuclear Factor Y, Genomics, Drought stress, Transcription factors

\section{Background}

Transcription factors (TFs) play an important role in regulating multiple physiological processes in all living organisms. In general, TFs can be divided into different families by their recognizable conserved domains, such as MYB [17], GRAS [38], NAC [51, 52] and BZIP [44, 45]. Similar protein sequences generally indicate that TF family members have similar biological functions, which facilitates the identification and analysis of unknown proteins in other species. For example, AtNF-YB1 can enhance resistance in Arabidopsis under drought conditions and an orthologous maize $N F-Y$ factor, ZmNF-YB2, was shown to have an equivalent activity in maize [28]. NUCLEAR FACTOR Y (NF-Y), also called heme-activated protein (HAP) or

\footnotetext{
* Correspondence: zan_hope@163.com

Shandong Institute of Pomology, Taian, Shandong, China
}

CCAAT binding factor $(\mathrm{CBF})$, is a heterotrimeric complex composed of three unique subunits: NF-YA, NF-YB, and NF-YC [25]. In the promoter regions, the NF-Y TF binds to cis-elements with the conserved core sequence CCAAT to activate or inhibit the expression of related functional genes in metabolism [25]. Three subfamilies (NF-YA, NF-YB, and NF-YC) can be characterized by their conserved domains and sequence lengths. In general, NF-YA sequences are longer than those of NF-YB and NF-YC. NF-YA has a core region composed of 53 amino acids, which contains two conserved domains, A1 and A2 [27]. NF-YB sequences are shorter than those of NF-YC. The protein structures of NF$\mathrm{YB}$ and NF-YC are similar to those of $\mathrm{H} 2 \mathrm{~B}$ and $\mathrm{H} 2 \mathrm{~A}$ histones, respectively [27]. Initially, NF-YB and NF-YC form a dimer in the cytoplasm. This dimer is transferred into the nucleus, where it interacts with NF-YA to complete the assembly of the heterotrimeric complex [39]. 
In recent years, the role of members of the $N F-Y$ gene family under drought stress has been a popular research topic. In several species, $N F-Y$ gene family members have been identified across their genomes and analysed under drought stress. In Citrus, a total of 22 CsNF- $Y$ genes have been identified and the candidate gene CsNF-YA5 was shown to exerted distinct effects on the dehydration tolerance of transgenic tobacco [33]. In castor bean, 25 $R c N F-Y$ genes have been identified across the genome and their expression changes were investigated under four types of abiotic stresses (drought, cold, heat and salt stresses) [44, 45]. In chickpea, a total of $40 \mathrm{CaNF-Y}$ genes have been identified and some CaNF-Y genes were found to be responsive to dehydration and abscisic acid treatments [9].

Peach (Prunus persica L.) is one of the most popular fruits worldwide. Because of its small genome size, economic and nutritional importance, and short reproductive cycle [7], peach has become a model tree species for plant physiology and genetics research. With the release of the whole genome sequence of peach [15], it has become convenient to analyse entire gene families. The $N F-Y$ gene family plays vital roles in various physiological processes and regulatory networks. However, a detailed analysis of the peach $N F-Y$ (designated $P p N F-Y$ ) gene family has not yet been performed. Considering all these points, the aim of this study was to understand the members of the $N F-Y$ gene family in peach and explore the roles of these genes under drought stress. In this study, based on the sequence information derived from public databases, $P p N F-Y$ members were identified and classified. Gene duplication was analysed to identify the evolutionary origins of $P p N F-Y$ gene family. In addition, the current study analysed the phylogenetic relationships of NF-Y proteins in peach and Arabidopsis to further elucidate the biological functions of $P p N F-Y$ genes. Tissue expression profiles were examined to further investigate the roles of PpNF-Y genes during the development of different organs. Moreover, expression analysis during drought stress treatment indicated that some $P p N F-Y$ genes responded to drought stress and these genes served as candidate genes for drought resistance in peach.

\section{Methods}

\section{Plant materials and drought treatments}

Prunus davidiana seeds (Pd) were collected from the Jinniu Mountain Scientific Research base of Shandong Institute of Pomology, Shandong, China, located at $35^{\circ} 38^{\prime} \mathrm{N}$ and $116^{\circ} 20^{\prime} \mathrm{E}$. The seeds were grown in plastic pots (upper diameter $7.0 \mathrm{~cm}$, lower diameter $5.0 \mathrm{~cm}$, height $7.8 \mathrm{~cm}$ ) filled with mixed soil (vermiculite:humus $=1: 1$ ) (without chemical fertilizer application) and were well watered for $30 \mathrm{~d}$ under greenhouse conditions with
$24 / 18^{\circ} \mathrm{C}$ day/night, $16 / 8 \mathrm{~h}$ light/dark and $70 \%$ relative humidity [32]. In the experiment, there were two groups, a control group and a treated group. Each of them contained 10 peach seedlings. The drought stress treatments were carried out on ten Pd seedlings with similar stem lengths and leaf areas for ten days by withholding water until distinct wrinkling was observed in the top three leaves [29]. The control pot contained another ten similar Pd seedlings that were slightly watered every three days to keep the soil moist. Leaf samples from the treated and control groups were collected, immediately frozen in liquid nitrogen and stored at $-70^{\circ} \mathrm{C}$ [1]. In the tissue expression pattern experiment, samples of five tissues including roots, stems, leaves, flowers and fruits were collected from the cultivar Sunagowase, immediately frozen in liquid nitrogen and stored at $-70^{\circ} \mathrm{C}[1]$.

\section{Identification and analysis of PpNF-Ys}

Siefers et al. [37] described the conserved regions of the three NF-Y subfamilies: the NF-YA conserved region contains the amino acid sequence f-V-N-A-K-Q-Y-h-X-I-l-rR-R-q-x-R-A-k-l-E-a-X-x-K-l-i-k-x- R-K-P-Y-l-H-E-S-R-H$x-H-A-x-r-R-p-R-G-s-G-G-R-E$, the NF-YB conserved region contains the amino acid sequence $r-e-q-D-r-x-L-P-I-$ A- N-v-x-R-I-M-K-x-x-L-P-x-x-n-x-k-i-s-k-D-A-K-e-t-xQ-E-C-v-s-E-F-I-S-F-v-T-s-E-A-s-d-k-C-q-x-E-k-R-K-T-In-g-d-D-x-L-w-A-m-x-t-L-G-F-x-d-Y-x-e-p-L-x-k-x-Y-x$\mathrm{L}-\mathrm{x}-\mathrm{k}-\mathrm{y}-\mathrm{R}-\mathrm{e}-\mathrm{x}-\mathrm{x}-\mathrm{e}-\mathrm{g}-\mathrm{e}$, and the NF-YC conserved region contains the amino acid sequence l-P-l-a-R-I-K-K-I-M- Kx-D-e-D-V-x-m-I-s-a-e-A-P-x-l-f-a-K-A-c-E-M-F-I-x-e-LT-x-R-s-W-x-h-t-e-e-n-k-R-r-T-l-q-k-x-d-i-a-a-A-v-x-r-x$\mathrm{d}-\mathrm{x}-\mathrm{x}-\mathrm{f}-\mathrm{D}-\mathrm{F}-\mathrm{L}-\mathrm{x}-\mathrm{x}-\mathrm{D}-\mathrm{x}-\mathrm{V}-\mathrm{P}$, where the uppercase letters represent completely conserved sites, the lowercase letters except $\mathrm{x}$ represent relatively conserved sites, and the lowercase $\mathrm{x}$ represents non-conserved sites. This study characterized 24 NF-Y family members in peach by using the above three conserved regions to blast the peach protein database (version 2.0, https://www.rosaceae.org/blast/protein/protein) [16]. The candidate $N F-Y$ genes were considered to be PpNF-Ys. The online program Conserved Domains (https://www.ncbi.nlm.nih.gov/Structure/) was used to ensure the conserved domains of the candidate NF-Ys [24]. PpNF-Y conserved motifs were identified using the MEME program (http://www. meme. sdsc.edu/ meme/meme.html) [42].

\section{PpNF-Y sequence structure and genome distribution}

The distribution of gene exons and introns was analysed using Gene Structure Display Server 2.0 software (http:// gsds.cbi.pku.edu.cn/index.php) [14]. The detailed structures of the genes were drawn with Illustrator for Biological Sequences software (Version 1.0.3; http://ibs.biocuckoo.org/ index.php) [22]. The locations of the PpNF-Ys on the genome were collected from a database (Prunus persica v2.1) 
in JGI (https://phytozome.jgi.doe.gov/jbrowse/index.html) [31]. Their genome distribution was displayed using the MapInspect tool (http://mapinspect.software.informer.com) [10].

\section{Calculating the parameters of NF-Y gene duplication events}

The paralogous nucleotide sequences were pairwise aligned by MEGA 5.0 [40]. The parameters Ks (synonymous substitution rate) and $\mathrm{Ka}$ (nonsynonymous substitution rate) were calculated by the program DNASP 6.0 [34]. The date $(T)$ of the duplication events was estimated by the formula $T=\mathrm{Ks} / 2 \lambda$, where $\lambda$ represents the estimated clock-like rate of synonymous substitution, which was $1.5 \times 10^{-8}$ substitutions/synonymous site/year in dicots $[4,6]$.

PpNF-Y sequence alignment and phylogenetic analysis The PpNF-Y protein sequence alignments were analysed using MEGA 5.0 software (https://www.megasoftware. net) [40] and the conserved domains were marked using GeneDoc 2.7.000 software [30]. A phylogenetic tree of PpNF-Y family proteins was constructed using the neighbour-joining (NJ) method [35] of MEGA 5.0 software with a bootstrap test $(n=1000)$.

\section{NF-Y protein sequences of Arabidopsis}

The Arabidopsis NF-Y gene family was identified and numbered in a previous study [37]. The gene sequences and the corresponding protein sequences were collected by searching the gene names such as NF-YA1 from the Arabidopsis Information Resource (TAIR) database (https://www.arabidopsis.org/) [3]. Arabidopsis NF-Y proteins were designated AtNF-Ys.

\section{Drought-responsive cis-elements of the PpNF-Y promoter} The promoter sequences (length, $1.5 \mathrm{~kb}$ ) of $P p N F-Y_{\mathrm{S}}$ were collected from the Genome Database for Rosaceae (https://www.rosaceae.org/). Drought-responsive cis-elements were analysed in the online software PlantCARE (http://bioinformatics.psb.ugent.be/webtools/plantcare/ html/).

The models of cis-elements in the promoters were made with Illustrator for Biological Sequences software [22].

\section{RNA isolation and expression analysis}

Total RNA from different tissues of Prunus davidiana (Pd) was extracted using $\mathrm{TRIzol}^{\circ}$ reagent (Invitrogen Inc., USA). According to the supplier's protocol, the extracted RNA was used as a template with M-MLV reverse transcriptase (Toyobo, Japan) to synthesize complementary DNA (cDNA). Real-time PCR was performed using a 7500 Fast Real Time PCR System (Applied Biosystems, NY, USA). One microlitre cDNA template, $10 \mu \mathrm{L}$ SYBR Premix Ex
Taq (Takara, Kyoto, Japan), $0.8 \mu \mathrm{L}$ gene-specific primers, and $8.2 \mu \mathrm{L} \mathrm{ddH}_{2} \mathrm{O}$ were mixed to compose $20 \mu \mathrm{L}$ reaction systems. The PCR thermal cycle was as follows: $95^{\circ} \mathrm{C}$ for 30 s; 40 cycles of $95^{\circ} \mathrm{C}$ for $5 \mathrm{~s}, 52^{\circ} \mathrm{C}$ for $30 \mathrm{~s}$, and $72^{\circ} \mathrm{C}$ for $20 \mathrm{~s}$. Translation elongation factor 2 (TEF2) was used as an internal control to normalize gene expression [2, 43]. Each sample was analysed with three biological replicates.

\section{Statistical analysis}

All data were subjected to analysis of variance according to Student's $t$ test using SPSS statistical software 17.0 (SPSS Inc., USA) [21].

\section{Result}

\section{Identification of PpNF-Y family members}

After removing different transcripts of the same gene, a total of 24 non-redundant protein sequences (6 PpNF-YAs, 12 PpNF-YBs and 6 PpNF-YCs) representing the primary transcript were identified. All 24 PpNF-Ys contained one highly conserved domain of the three NF-Y subfamilies that were also confirmed by the online database of Conserved Domains. To distinguish these newly identified genes, the current study renamed these genes based on subfamily branch and chromosomal distribution (PpNF-YA1-PpNFYA6, PpNF-YB1-PpNF-YB12 and PpNF-YC1-PpNF-YC6). The protein lengths of the 24 PpNF-Ys ranged from 121 AA to 398 AA (Table 1), showing a wide distribution of PpNF-Y lengths. Among the three subfamilies of PpNF-Y, the PpNF-YA lengths (range from 202 AA to 398 AA, average length $322.5 \mathrm{AA}$ ) were generally longest, the PpNF-YC lengths (range from $121 \mathrm{AA}$ to $280 \mathrm{AA}$, average length 228.5 AA) were shorter, and the PpNF-YB lengths (range from 167 AA to 254 AA, average length 203.2 AA) were shortest. The predicted molecular weights $(M w)$ of the 24 PpNF-Ys ranged from 13.26 (PpNF-YC4) to 44.05 (PpNFYA4), and the predicted theoretical isoelectric points $(p I)$ of the 24 PpNF-Ys ranged from 5.27 (PpNF-YB2) to 9.43 (PpNF-YA5).

\section{Phylogenetic relationships, genome distribution and gene structure of PpNF-Ys}

To investigate the phylogenetic relationships among the $P p N F-Y_{\mathrm{s}}$, three phylogenetic trees were constructed based on an alignment of the PpNF-Y nucleotide sequences (Additional file 1) using MEGA 5.0 (Fig. 1). The neighbour-joining $(\mathrm{NJ})$ phylogenetic trees were constructed to show the structural classification of the PpNF-Ys. As shown in Fig. 1, the NJ tree was distinctly divided into three subgroups, marked by three different colours (PpNF-YAs, red; PpNF$Y B$ s, green; $P p N F-Y C$ s, blue), which were consistent with our identification results. The $P p N F-Y A$ branch had a simple structure, and a pair of paralogous PpNF-YAs (PpNF-YA2 and 6) was detected. In the $P p N F-Y B$ branch, there were four major groups. A group containing $P p N F-Y B 2$ and 10 
Table 1 Information of the NF-Y genes family in peach

\begin{tabular}{|c|c|c|c|c|c|c|c|c|}
\hline Name & Gene & Chromosome localization & CDS & Length (AA) & $\mathrm{Mw}(\mathrm{kD})$ & $\mathrm{Pl}$ & $\begin{array}{l}\text { Homologs } \\
\text { in Arabidopsis }\end{array}$ & $\begin{array}{l}\text { Functional annotation } \\
\text { in Arabidopsis }\end{array}$ \\
\hline PpNF-YA1 & Prupe.2G014500 & Pp02:1285333..1293724+ & 1035 & 344 & 37.66 & 8.32 & AtNF-YA1,7 & Salt Stress [21] \\
\hline PpNF-YA2 & Prupe.3G043900 & Pp03:3121596..3127378+ & 1032 & 343 & 38.00 & 9.20 & AtNF-YA3,8 & Embryo development [48] \\
\hline PpNF-YA3 & Prupe.4G090200 & Pp04:4494268..4499893- & 609 & 202 & 22.13 & 7.90 & AtNF-YA4,7 & Flower [11] \\
\hline PpNF-YA4 & Prupe.4G249900 & Pp04:16710014..16714553+ & 1197 & 398 & 44.05 & 5.89 & AtNF-YA1,9 & Seed development [50] \\
\hline PpNF-YA5 & Prupe.7G093100 & Pp07:12566137..12572248+ & 966 & 321 & 35.35 & 9.43 & AtNF-YA2,10 & Seed germination [41] \\
\hline PPNF-YA6 & Prupe.7G203500 & Pp07:18875767..18879196+ & 984 & 327 & 36.37 & 8.81 & AtNF-YA3,8 & Embryo development [48] \\
\hline$P p N F-Y B 1$ & Prupe.1G138500 & Pp01:10829440..10830284+ & 492 & 163 & 18.55 & 5.62 & AtNF-YB5,7 & Unknown \\
\hline$P p N F-Y B 2$ & Prupe.2G219500 & Pp02:24828695..24832311+ & 543 & 180 & 19.19 & 5.27 & AtNF-YB1 & Drought stress [25] \\
\hline$P p N F-Y B 3$ & Prupe.3G197300 & Pp03:20770866..20771306- & 546 & 181 & 19.11 & 5.38 & AtNF-YB3 & Flowering, root growth [47] \\
\hline PpNF-YB4 & Prupe.4G003800 & Pp04:258372..261155- & 738 & 245 & 26.87 & 6.29 & AtNF-YBG & Embryonic desiccation-intolerance [18] \\
\hline PpNF-YB5 & Prupe.4G004200 & Pp04:271278..272705- & 765 & 254 & 29.78 & 9.00 & AtNF-YB3 & Flowering, root growth [47] \\
\hline PpNF-YB6 & Prupe.4G242700 & Pp04:16019863..16021262+ & 600 & 199 & 21.41 & 6.66 & AtNF-YB3 & Flowering, root growth [47] \\
\hline$P p N F-Y B 7$ & Prupe.4G243500 & Pp04:16087429..16090365- & 750 & 249 & 26.72 & 6.44 & AtNF-YB9 & Embryonic desiccation-intolerance [18] \\
\hline PpNF-YB8 & Prupe.4G273800 & Pp04:22774061..22774753- & 693 & 230 & 25.92 & 6.20 & AtNF-YB6 & Embryonic desiccation-intolerance [18] \\
\hline PpNF-YB9 & Prupe.6G175100 & Pp06:17959164..17964486- & 651 & 216 & 24.17 & 5.77 & AtNF-YB3 & Flowering, root growth [47] \\
\hline PpNF-YB10 & Prupe.6G241800 & Pp06:24020199..24022272- & 522 & 173 & 18.69 & 6.15 & AtNF-YB8,10 & Unknown \\
\hline PpNF-YB11 & Prupe.6G323400 & Pp06:28504283..28505052+ & 519 & 172 & 19.11 & 5.41 & AtNF-YB4,5 & Unknown \\
\hline PpNF-YB12 & Prupe.8G256000 & Pp08:21852595..21853514+ & 531 & 176 & 19.91 & 6.66 & AtNF-YB5 & Unknown \\
\hline PpNF-YC1 & Prupe.1G152500 & Pp01:12042456..12046186+ & 783 & 260 & 28.87 & 5.89 & AtNF-YC9 & Flowering [37] \\
\hline$P p N F-Y C 2$ & Prupe.3G149300 & Pp03:16308590..16312026+ & 636 & 211 & 24.53 & 5.37 & AtNF-YC2,3 & Flowering [37] \\
\hline PpNF-YC3 & Prupe.4G132200 & Pp04:7318206..7321121+ & 843 & 280 & 31.40 & 5.76 & AtNF-YC2,3 & Flowering [37] \\
\hline PpNF-YC4 & Prupe.5G096000 & Pp05:10523596..10524073+ & 366 & 121 & 13.26 & 7.82 & AtNF-YC3,9 & Flowering [37] \\
\hline PpNF-YC5 & Prupe.5G150400 & Pp05:13652222..13654960+ & 714 & 237 & 26.04 & 5.31 & AtNF-YC4 & Floral induction [45] \\
\hline PpNF-YC6 & Prupe.6G328500 & Pp06:28759728..28761956- & 789 & 262 & 29.16 & 5.96 & AtNF-YC3,9 & Flowering [37] \\
\hline
\end{tabular}

was located outside the three other groups. The presence of a bifurcation put PpNF-YB12 outside a secondary subgroup formed by $P p N F-Y B 1,9$ and 11. The third group was composed of $P p N F-Y B 4$ and 8 . In the fourth group, there were also two bifurcations separating $P p N F-Y B 3$ and 7 from a second subgroup composed of PpNF-YB5 and 6. The branch structure of the PpNF-YCs was similar to that of the PpNF-YAs. A pair of paralogous PpNF-YCs (PpNF-YC3 and $5)$ was located on the innermost side, and the remaining $P p N F-Y C$ s were located around the outside.

Twenty-four $P p N F-Y_{\mathrm{s}}$ were distributed on all eight chromosomes (Fig. 2). Most PpNF-Ys with eight members were distributed on the 4 th chromosome. The fewest of PpNF-Ys were located on the 8th chromosome, with only one member. The other six chromosomes contained 2-4 PpNF-Ys. The exons and introns of the $P p N F-Y_{\mathrm{s}}$ were drawn with Illustrator for Biological Sequences (IBS, version 1.0.3) software using the genomic DNA sequences of the $P p N F-Y_{\mathrm{S}}$ and the corresponding coding sequence (Fig. 3). All $P p N F-Y A s$ contained 4-6 introns. Some PpNF-YBs (PpNF-YB1, 4,
$6,8,11,12)$ and PpNF-YCs $(P p N F-Y C 1,3,4,6)$ were composed of only one exon.

\section{Duplication events of the NF-Y genes in the peach genome}

In the phylogenetic analysis of the NF-Y gene family, 6 pairs of paralogous $N F-Y$ genes were detected (Fig. 2). Among them, 3 pairs (PpNF-YA2 and PpNF-YA6, $P p N F-Y B 2$ and PpNF-YB10, PpNF-YC3 and PpNF$Y C 5)$ were randomly scattered on different chromosomes, and the other three pairs (PpNF-YB5 and $P p N F-Y B 6, P p N F-Y B 4$ and PpNF-YB8, PpNF-YB9 and $P p N F-Y B 11)$ were located on the same chromosomes. To further trace the dates of the duplication events, the parameters $\mathrm{Ks}$ and $\mathrm{Ka}$ and the $\mathrm{Ka} / \mathrm{Ks}$ ratio were estimated using DNASP 6.0 software [34]. The Ka/Ks ratios of $P p N F-Y B 9$ and $P p N F-Y B 11$ were greater than 1 and those of the other five pairs of paralogous $P p N F-Y_{\mathrm{S}}$ were less than 1 . The approximate dates of the duplication events are shown in Table 2. The origin dates of the three pairs of paralogous $P p N F-Y s$ on 




Fig. 1 The phylogenetic relationship of the PpNF-Y gene family

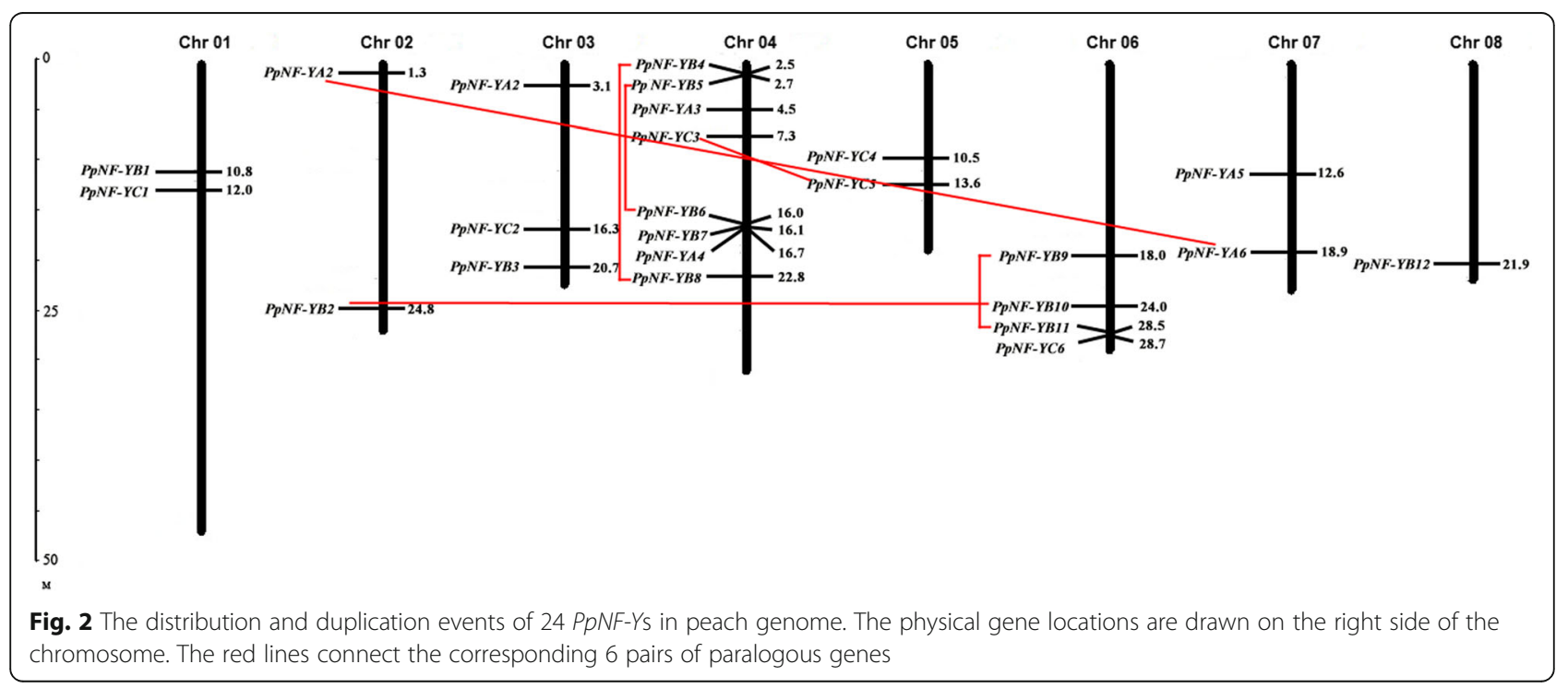




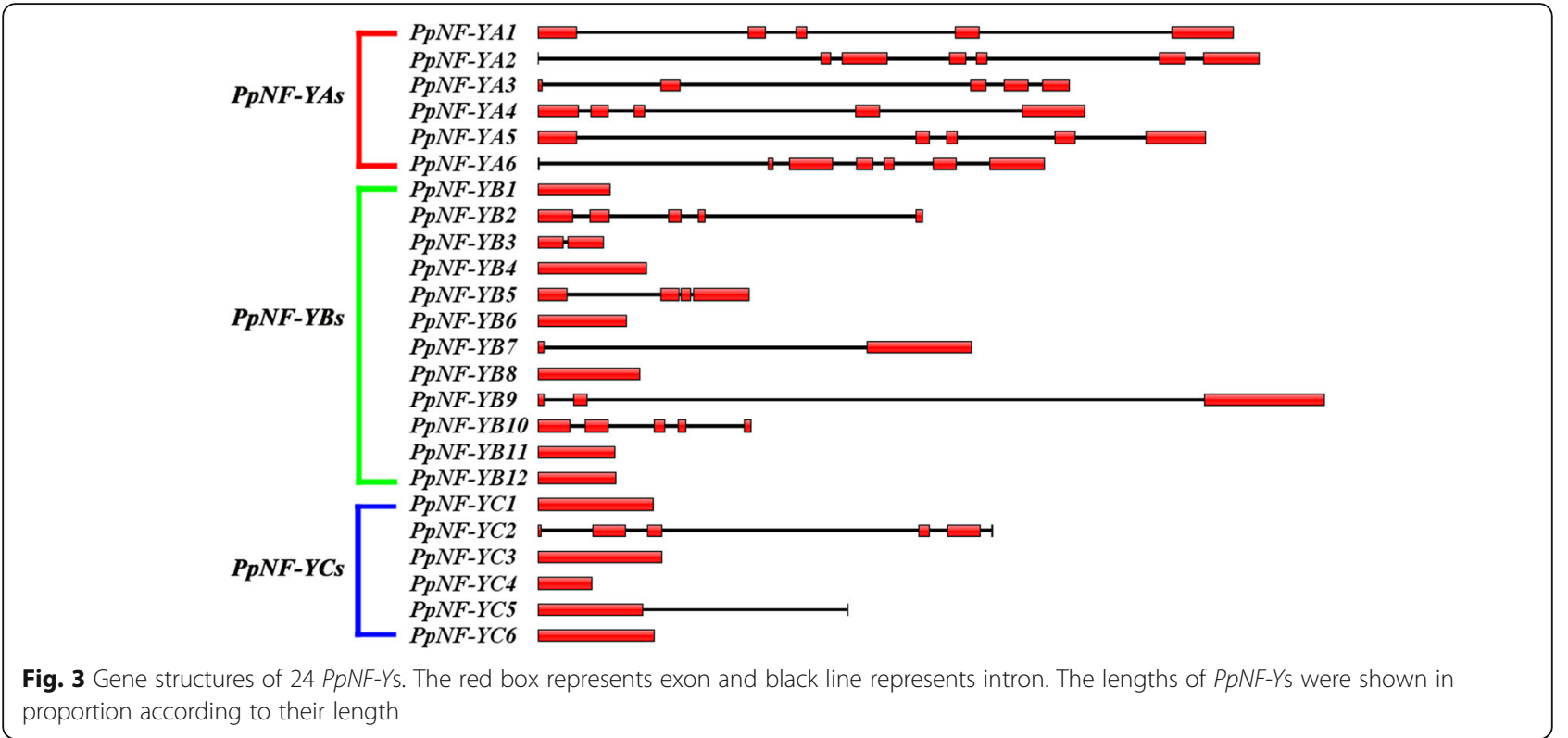

the different chromosomes ranged from 20.6 to 59.67 million years ago. The dates of the other three pairs on the same chromosomes ranged from 13.33 to 68.33 million years ago.

\section{Conserved regions of PpNF-Ys}

To further investigate the conserved regions of the three subfamilies in peach, multiple protein sequence alignments of the PpNF-YAs, PpNF-YBs, and PpNF-YCs were analysed using MEGA 5.0. All three subfamilies contained conserved regions. Multiple alignment of the PpNF-YA proteins indicated that there was a conserved region composed of approximately 50 amino acids (Fig. 4a). As with the PpNF-YAs, two conserved regions of PpNF-YB (approximately 102 amino acids) and PpNF-YC (approximately 81 amino acids) were identified by protein sequence alignment (Fig. 4b and c).

Table 2 The parameters and dates of the duplication evens in the paralogous PpNF-Ys.

\begin{tabular}{llllll}
\hline \multicolumn{2}{l}{ The paralogous gene } & KS & KA & $\begin{array}{l}\text { Ka/Ks } \\
\text { ratio }\end{array}$ & $\begin{array}{l}\text { Date (million } \\
\text { year ago) }\end{array}$ \\
\hline PpNF-YA2 & PpNF-YA6 & 0.66 & 0.55 & 0.83 & 22.00 \\
PpNF-YB5 & PpNF-YB6 & 1.08 & 0.21 & 0.19 & 36.00 \\
PpNF-YB4 & PpNF-YB8 & 2.05 & 0.43 & 0.21 & 68.33 \\
PpNF-YB9 & PpNF-YB11 & 0.4 & 0.55 & 1.38 & 13.33 \\
PpNF-YB2 & PpNF-YB10 & 0.62 & 0.28 & 0.45 & 20.67 \\
$P p N F-Y C 3$ & $P p N F-Y C 5$ & 1.79 & 0.22 & 0.12 & 59.67 \\
\hline
\end{tabular}

Ks and Ka were calculated using the program DNASP 6.0 [34]. The date of the duplication events in the paralogous PpNF-Ys were analzyzed by the formula $T$ $=\mathrm{Ks} / 2 \lambda, \lambda$ representing the estimated clock-like rates of synonymous substitution, which was $1.5 \times 10^{-8}$ substitutions/synonymous site/year in dicots [4]
To reveal the putative motifs of the $N F-Y$ family in peach, 24 PpNF-Ys were analysed using the program MEME. All members contained three distinct motifs (Fig. 5), which was consistent with the fact that they belonged to the same gene family.

Expression patterns of PpNF-Ys in different peach organs The expression patterns of $P p N F-Y \mathrm{~s}$ in the five organs (roots, stems, leaves, flowers and fruits) were analysed (Fig. 6). The PpNF-Ys showed different expression patterns. Some were constitutively expressed in every organ such as $P p N F-Y B 5$, while others showed specific high expression in one or two peach organs, such as PpNF-YA4 (leaves and fruits), PpNF-YB6 (flowers) and PpNF-YB4 (stems). This diversity of expression patterns of $P p N F-Y_{\mathrm{s}}$ suggested a divergence in the biological functions of $P p N F-Y$ s during peach growth and development.

\section{Evolutionary relationships of the NF-Y family in peach and Arabidopsis}

The functions of some AtNF-Y family members have been identified in Arabidopsis. However, the biological functions of PpNF-Y proteins are unknown. To predict the functions of PpNF-Y members, we explored the phylogenetic relationships of the NF-Y members from Arabidopsis and peach using 60 NF-Y protein sequences (Fig. 7; Additional files 2 and 3). The NJ phylogenetic tree showed that 58 NF-Y members (excluding AtNF-YB11 and AtNFYC11) could be classified into three main groups distinguished by three different colours (red, green and blue), which were consistent with the subfamily classifications of the PpNF-Ys. In each group, we found that some pairs of paralogous NF-Y proteins were composed of one PpNF-Y 


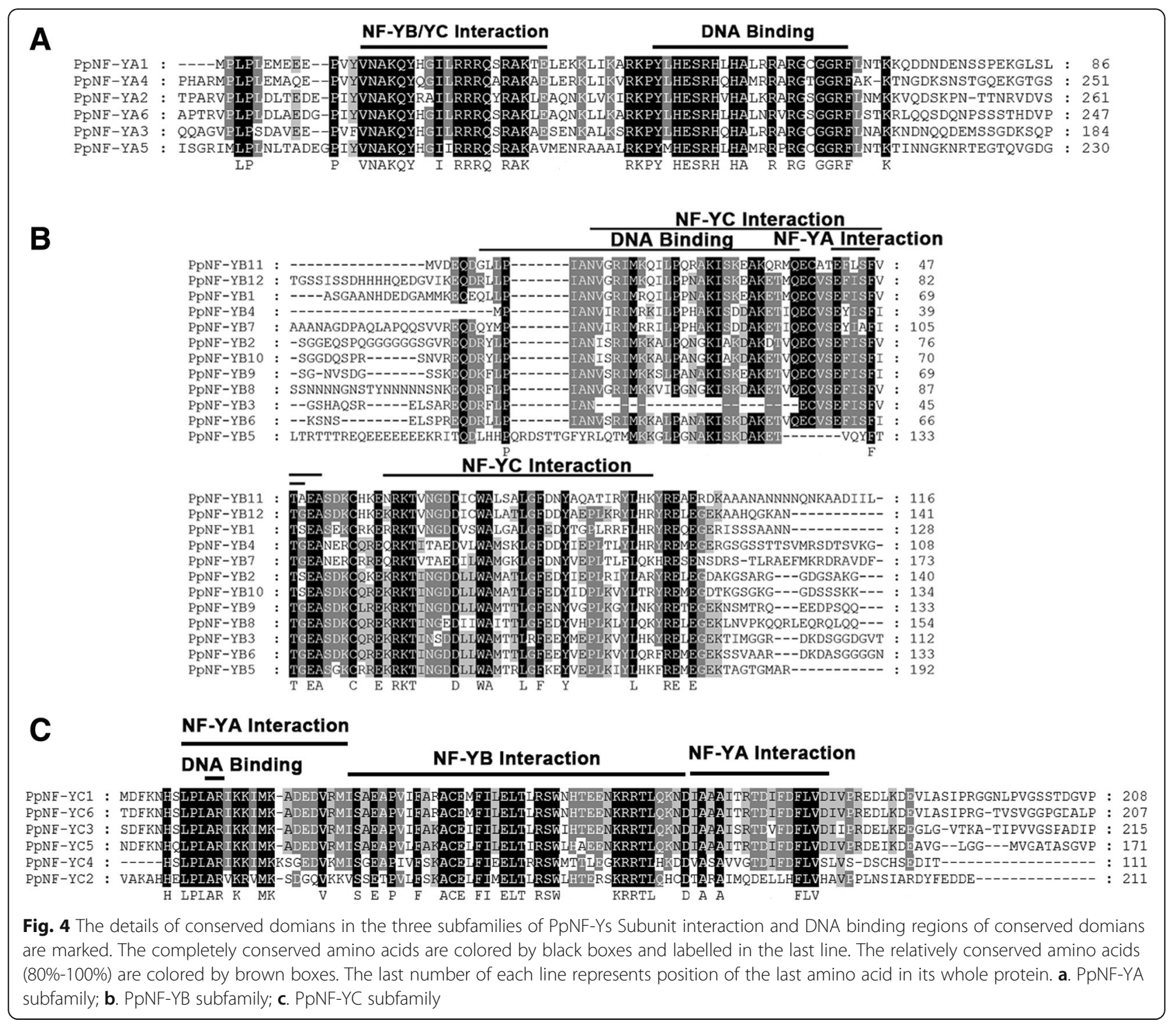

and one AtNF-Y, such as PpNF-YA4 and AtNF-YA9, and this close evolutionary relationship generally suggested the similarity of their biological functions. Both the NFYA and NF-YC groups contained one pair of paralogous NF-Y proteins, and the NF-YB group contained three pairs.

\section{Analysis of five drought-responsive cis-elements in the promoter sequences of $P p N F-Y$ genes}

To explore the involvement of the PpNF-Y genes in drought tolerance, their promoter sequences were analysed using PlantCARE software [19]. It is generally known that five cis-elements, ABREs, MBSs, G-boxs, W-boxs and DREs, respond to drought-induced signalling and regulation of downstream gene expression [18, 26, 36]. All five cis-elements in the promoter regions of the PpNF-Y gene family members are shown in Fig. 8. The results indicated that all
24 PpNF-Y promoter regions contained one or more drought-responsive cis-elements. ABREs, MBSs, G-boxes, W-boxes and DREs were distributed within 17, 9, 8, 10 and 7 PpNF-Y promoter regions, respectively. The numbers of drought-responsive cis-elements in the $24 P p N F-Y$ promoter regions ranged from 1 (PpNF-YC4) to 8 (PpNF-YB1), and the types ranged from 1 (PpNF-YC4) to 4 (PpNF-YB1).

\section{Expression analysis of $P p N F-Y s$ under drought stress}

Nelson et al. [28], Chen et al. [8] and Pereira et al. [33] have reported that the $N F-Y$ gene family is closely related to drought tolerance in some species, including Arabidopsis, Citrus, maize, and Bermuda grass. In peach, it is possible that one or more $P p N F-Y_{\mathrm{s}}$ are involved in tolerance to drought stress. To investigate drought-responsive $P p N F-Y_{\mathrm{s}}$, an expression analysis of the $24 P p N F-Y_{\mathrm{s}}$ from $\mathrm{Pd}$ seedlings under drought 


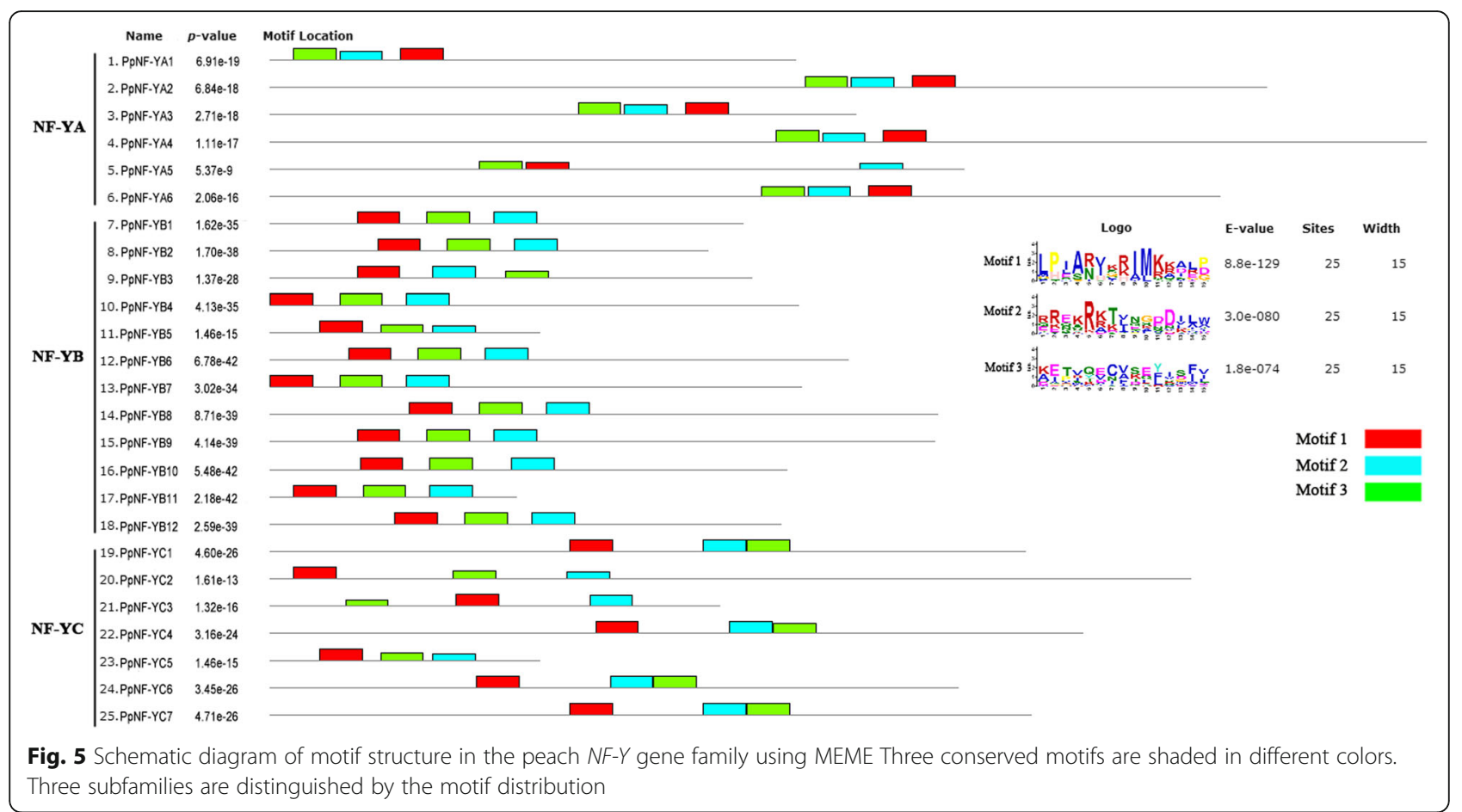

stress was performed (Fig. 9; Additional file 4). The results showed that $4 P p N F-Y A s$ (PpNF-YA3, 4, 5, and 6), 4 PpNF-YBs (PpNF-YB2, 6, 7, and 12) and PpNF$Y C 4$ were upregulated under drought stress. Among these members, the expression level of PpNF-YA5 increased the most, to approximately fifteen times that of the control sample. The upregulated gene PpNF$Y A 4$ and $P p N F-Y B 7$ showed the smallest increases in transcript abundance, less than two times that of the control. The increased expression of the other six upregulated $P p N F-Y$ s ranged from two to eight times that of the control.

\section{Discussion}

\section{Peach contains only 24 diverse NF-Ys}

The NF-Y TFs are found in all sequenced eukaryotes. This gene family has been studied in some higher plant species, such as maize, rice, tomato, and banana [20, 46, 47, 51, 52]. Mantovani [23] has revealed some properties of this family, including relatively conserved binding and interaction domains, three types of subunits, and diverse biological functions, which were beneficial to our research on the NF-Y gene family in peach. A total of 24 $N F-Y$ members were identified in peach, fewer in Arabidopsis (36 NF- $Y$ members), tomato (59 NF- $Y$ members), rice (34 NF- $Y$ members), and wheat (39 NF- $Y$ members). The small number of peach $N F-Y$ members may be associated with the small size of the peach genome. The 24 PpNF-Ys demonstrated diversity in several aspects, including protein length, gene structure, molecular weight, theoretical isoelectric point and expression pattern, which suggested a diversity of biological functions among PpNF-Ys.

\section{Putative segmental and tandem duplication events in the peach genome}

The paralogous genes distributed on different chromosomes are generally designated as segmental duplication events, and those co-located on the same chromosome are considered tandem duplication events [6]. In this study, the locations of 6 paralogous $N F-Y$ genes were either on the same chromosome or on different chromosomes, implying that both tandem and segmental duplication events contributed to the expansion of the $N F-Y$ genes in peach. When the $\mathrm{Ka} / \mathrm{Ks}$ ratios of the five paralogous $P p N F-Y_{\mathrm{s}}$ were less than 1, purifying selection of duplication events occurred and the corresponding paralogous PpNF-Y proteins were considered to be unchanged. In contrast, the $\mathrm{Ka} / \mathrm{Ks}$ ratios of $P p N F-Y B 9$ and $P p N F-Y B 11$ were greater than 1 , indicating that distinct variation between the PpNF-YB9 and PpNF-YB11 proteins occurred during the duplication event. As the initial point of peach evolution was still unclear, we could not determine whether the duplication events in this gene family predated the formation of the peach species. However, the oldest date of the duplication events among the 6 pairs of paralogous PpNF-Ys could be 68.33 million years ago, suggesting that this is an ancient gene family. At a minimum, it could indicate traces of peach evolution. 


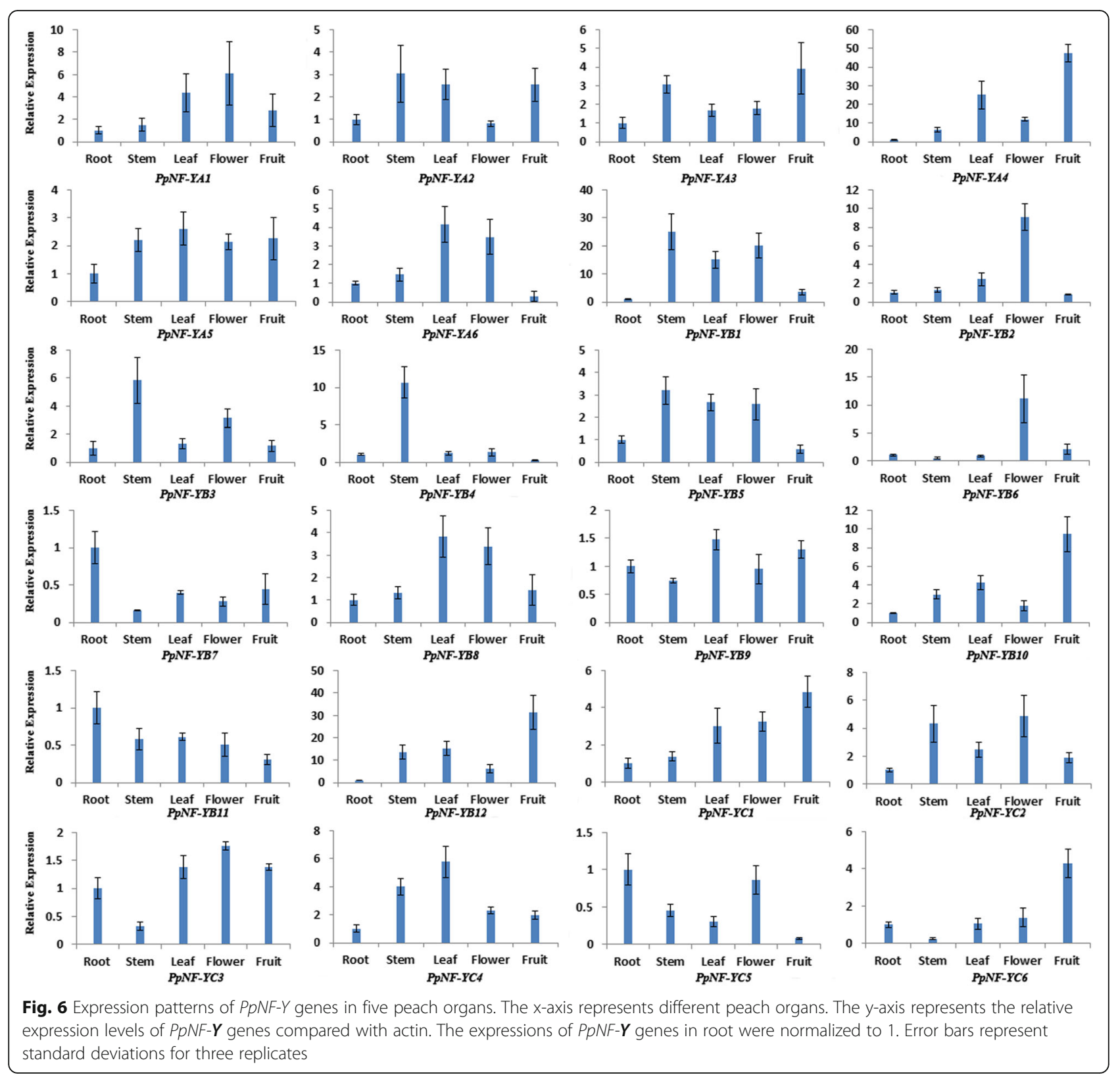

\section{Potential conserved domains in three NF-Y subfamilies}

In general, proteins can be characterized and classified by their conserved regions, which play vital roles in heterodimerization, heterotrimerization, and DNA interactions at CCAAT sites (Zambelli and Pavesi 2017). Previous studies have shown that all three subfamilies (NF-YA, NF-YB and NF-YC) are recognized by interaction domains that interact with NF-Y members and DNA-binding domains that bind to downstream targeted CCAAT sites [25]. Based on the recognized DNA-binding domain of NF-YA in plants, mammals and yeasts, the similar 17-amino-acid sequence Y-L-H-E-... -G-G-R$\mathrm{F}$ in the $\mathrm{C}$-terminus of the PpNF-YA conserved region was considered to interact with DNA at CCAAT sites
(Fig. 4a). It was inferred that the 21-amino-acid sequence Y-V-N-A- ... -A-K-L-E in the N-terminus of the PpNF-YA conserved region interacted with the other two subunits (PpNF-YB and PpNF-YC) (Fig. 4a).

The structure and amino acid composition of the NF$\mathrm{YB}$ conserved regions were similar to those of $\mathrm{H} 2 \mathrm{~B}$ histone fold motifs [12]. Based on the conserved regions of NF-YB members in Arabidopsis, a 31-amino-acid sequence R-x-L-P-... -E-T-x-Q was considered to be the DNA-binding domain of PpNF-YB. Similarly, two core regions, the 40-amino-acid sequence A-N-V-x-... -T-x$\mathrm{E}-\mathrm{A}$ and the 32 -amino acid sequence $\mathrm{x}-\mathrm{R}-\mathrm{K}-\mathrm{T}-\ldots . \mathrm{Y}-\mathrm{L}-\mathrm{x}-$ $\mathrm{X}$, were considered to interact with the other two subunits (PpNF-YA and PpNF-YC) (Fig. 4b). 


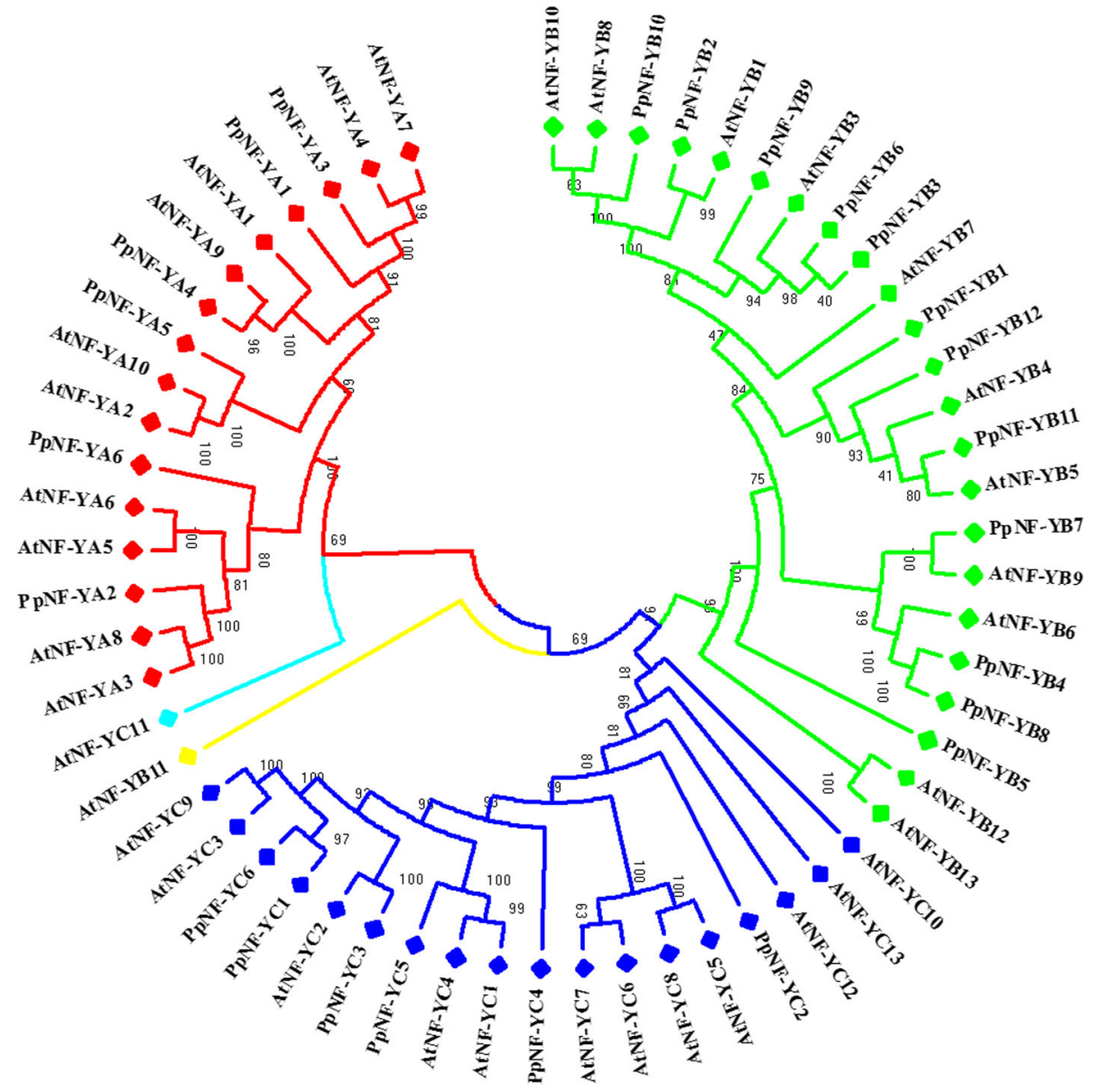

Fig. 7 Phylogenetic relationships of NF-Y proteins in peach and Arabidopsis Three subfamilies are shown in different colored

In the alignment analysis of 7 PpNF-YC members, the conserved 74-amino-acid sequence L-P-L-A-... -D-F-L$\mathrm{V}$ had similarities to an Arabidopsis subunit interaction domain (Fig. 4c). Thus, we inferred that this fragment was the core region by which PpNF-YC associated PpNF-YA/PpNF-YB. In addition, the DNA- binding domain of PpNF-YC was composed of only two residues in series "A" and "R", which were necessary for the formation of a complex between heterotrimeric NF-Y and DNA.

\section{Similar core protein regions between PpNF-Ys and AtNF- Ys imply similar biological functions}

Based on the analysis of the protein alignment, the conserved core amino acids of the PpNF-YA interaction and DNA-binding domains were almost highly consistent with those of AtNF-YAs, RnCFB-B (Rn, Rattus norvegicus), ScHAP2 (Sc, Saccharomyces cerevisiae), indicating that there may be similar biological functions of several $N F-Y A$ members among plants, mammals and yeasts. For example, most AtNF-YAs increased their expression under drought stress in Arabidopsis [13], and two-thirds of $P p N F-Y A$ s were also upregulated under drought stress in this study.

Boulard et al. [5] showed that the substitution of a required amino acid residue (from Lys to Asp) caused LEC1 (AtNF-YB9) and LEC1-like (AtNF-YB6) to differ from plant and animal NF-YB proteins, and this variation caused LEC1 to fail to rescue the lec1 embryonic desiccation-intolerant phenotype. The alternating distribution of PpNF-Y and AtNF-Y proteins in the NJ tree implied that some NF-Y members from these two species might originate from common ancestors, which might be reflected in their protein sequences. For example, we found that two PpNF-YB proteins, PpNF-YB4 and PpNF-YB7, were closely related to AtNF-YB9 and AtNF-YB6, and these four members together formed a subgroup in the NF-YB branch, which might suggest a similar biological function for them. Specifically, a substitution of required amino-acid residues (from Lys to Asp; the column marked by *) emerged in PpNF-YB4 and PpNF-YB7 (Fig. 4b), indicating that these two PpNF-YB members may have similar biological functions to those of AtNF-YB9 and AtNF-YB6. In Arabidopsis, 


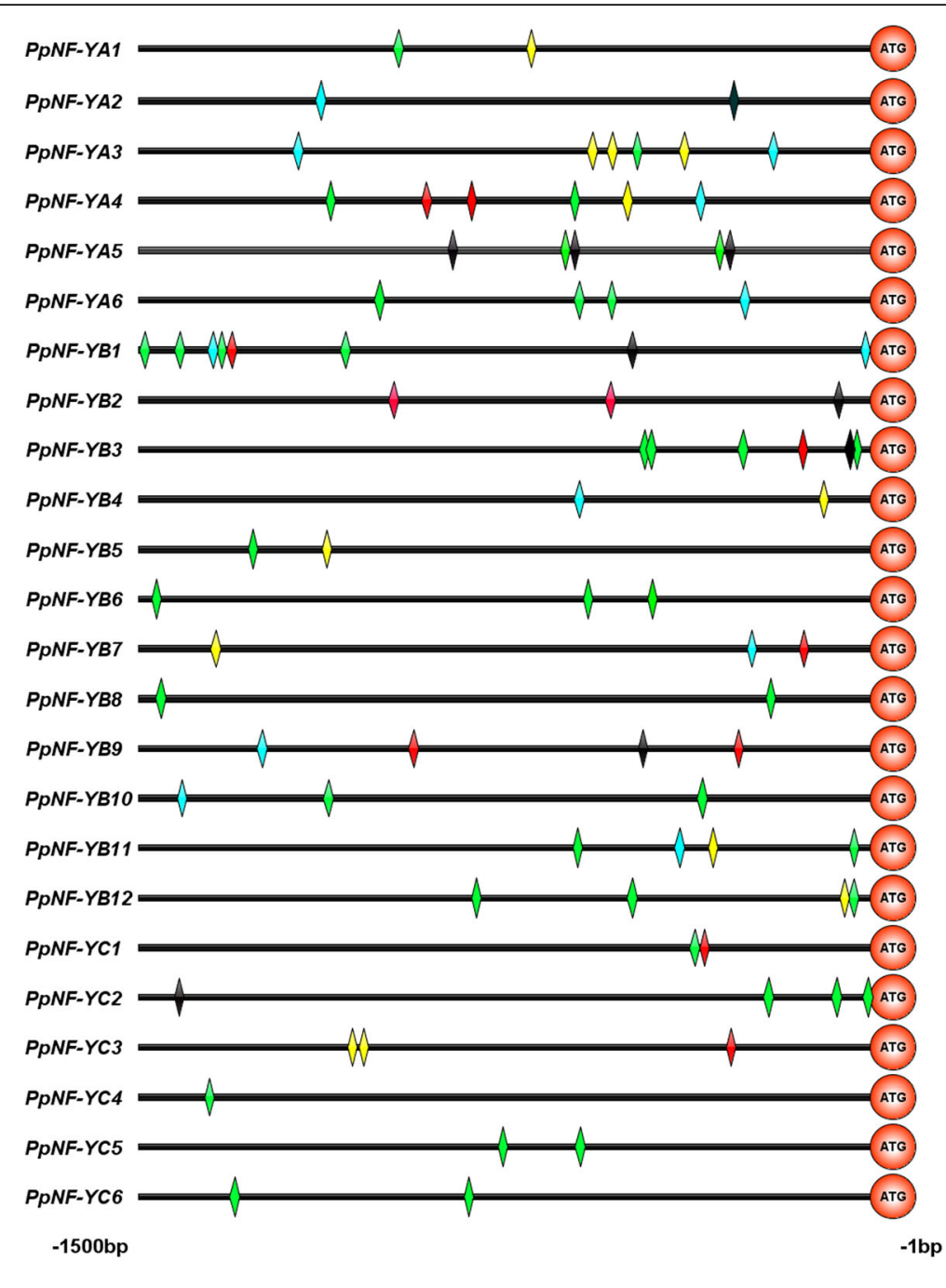

\section{$\triangle$ ABRE $\downarrow$ MBS $\neg$ G-box $\downarrow$ W-box $\$$ DRE}

Fig. 8 Schematic model of five drought-responsive cis-elements in the promoter sequences of PpNF-Y genes. MBS represents MYB binding site involved in drought-inducibility cis-element; ABRE represents cis-acting element involved in the abscisic acid responsiveness; DRE represents dehydration responsive element

AtNF-YB members have been shown to have biological functions in various developmental processes, such as stimulating cell division and expansion, promoting flowering, and synthetizing chloroplasts [39]. Based on the phylogenetic relationships of NF-Y proteins between Arabidopsis and peach, more biological functions of PpNF-YB members could be identified in various developmental processes.

\section{Diverse expression patterns of PpNF-Ys indicated a diversity of biological functions}

As gene expression patterns can provide important clues for gene function, the current study analysed the expression patterns of $P p N F-Y \mathrm{~s}$ in five peach organs. Some
$P p N F-Y$ s such as $P p N F-Y B$ and $P p N F-Y B 7$, were highly expressed in vegetative tissues, indicating that they might be involved in vegetative growth. Some $P p N F-Y_{\mathrm{s}}$ such as $P p N F-Y C 1$, were highly expressed in both vegetative tissues and reproductive tissues, showing that they might play multiple roles in the developmental process. However, some PpNF-Ys such as PpNF-YB10 and PpNF$Y C 6$, were specifically expressed in reproductive tissues, implying that they might participate in the development of floral organs and fruits. The expression patterns of $P p N F-Y_{\mathrm{s}}$ can reveal the behaviours of $P p N F-Y_{\mathrm{s}}$ during peach growth and development, providing useful information for further identification of the biological functions of $P p N F-Y$ s. 


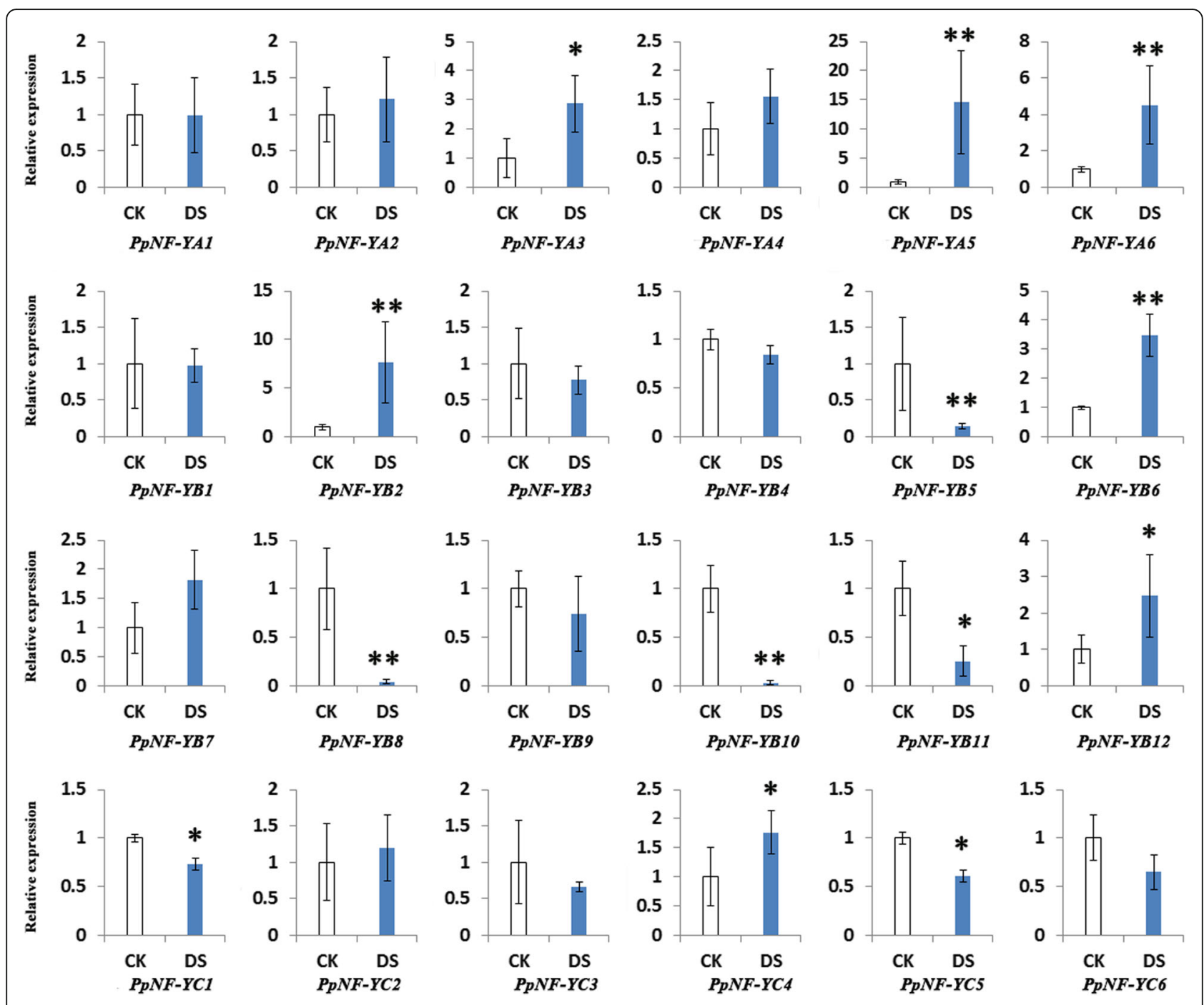

Fig. 9 Relative expression analysis of the 24 PpNF-Ys from Pd (Prunus davidiana) seedlings under drought stress was performed. Data presented are the means \pm SD of three replicates. CK represents seedlings without drought stress. DS represents seedlings under drought stress

\section{PpNF-Ys as candidate drought-tolerance genes}

The NF- $Y$ TFs are closely related to drought stress tolerance. NF-Y members, including Arabidopsis NF-YA5 and $N F-Y B 1$, maize $N F-Y B 2$, soybean $N F-Y A 3$, poplar $N F$ $Y B 7$, and bermudagrass $N F-Y C 1$, have been identified to participate in tolerance to drought stress [8, 29, 49]. Moreover, the transcriptional expression of drought-tolerance genes is generally induced by drought stress. This character could be used for identifying unknown drought-tolerance genes in peach.

The analysis of drought-responsive cis-elements in the $P p N F-Y$ promoter sequences implied that some members might be involved in the drought-responsive pathway. The $P p N F-Y$ promoter regions containing multiple types of drought-responsive cis-elements suggested that PpNF-YS might be involved in different drought-responsive pathways. Based on an analysis of gene expression under drought stress, 9 upregulated $P p N F-Y$ s could serve as candidate genes to analyse drought tolerance in peach. The NJ tree of PpNF-Y and AtNF-Y proteins showed a close relationship between AtNF-YB1 and PpNF-YB2, which was consistent with our inference that $P p N F-Y B 2$ might be a drought-resistance gene, similar to AtNF-YB1. PpNF-YA5, another candidate drought-resistance gene, also showed a close relationship with the drought-resistance gene AtNF-YA5, supporting our analysis and prediction. This study provided the possibility to further research the novel drought-tolerance pathway related to the $N F-Y$ genes in peach.

The NF- $Y$ gene family is a popular research topic

The strong correlation between the NF-Y gene family and drought resistance has become a popular research topic and has been demonstrated in many species. Recently, in Citrus, castor bean and chickpea, NF-Y gene 
family has been identified and candidate drought resistance genes members were analysed. Compared with recent published studies, this study contained some unique insights. First, the analysis of the PpNF-Y gene duplication indicated that most gene duplication events occurred long ago and generated similar duplicate genes, providing important clues into the evolutionary origins of the PpNF-Y gene family. Second, the details of drought-responsive cis-elements in the $P p N F-Y$ promoters were shown, which is useful for exploring the upstream elements of the PpNF- $Y$ genes involved in the drought resistance pathway. Third, the results of the phylogenetic relationships of $N F-Y$ proteins between Arabidopsis and peach suggested that a continued targeted functional analysis of the $P p N F-Y$ genes could be performed, such as for PpNF-YB2-AtNF-YB1 (drought resistance) and PpNF-YB4-AtNF-YB9 (desiccation-intolerant phenotype).

\section{Conclusions}

Since the NF- $Y$ gene family was first recognized and classified, many studies have focused on this gene family. Recently, published studies have identified the $N F-Y$ gene family in various plant species including maize, tomato, rice, banana and analysed the functions of this gene family members in some processes such as fruit ripening and abiotic stress. The current study found that $N F-Y$ gene family members were frequently identified in field crop and vegetable crop species, and this research trend has been extended to fruit trees. Our study, carried out in peach, closely followed the current research focus. Twenty-four $P p N F-Y$ s were first identified in the peach genome in the current study. Each subfamily of the $P p N F-Y_{\mathrm{s}}$ contained typical characteristics. The analysis of the duplication events clearly displayed the expansion of $P p N F-Y \mathrm{~s}$ across the genome. The current study indicated that there were some structural similarities of $N F-Y_{\mathrm{S}}$ between peach and Arabidopsis. The reported AtNF-YS were useful to predict and analyse the biological functions of the peach $N F-Y$ gene family. In particular, the current study predicted the functions of some PpNF-Ys such as PpNF-YBA-AtNF-YB9, PpNF-YB2-AtNF-YB1, with the help of similar conserved domains and close evolutionary relationships to the reported AtNF-Ys, which allowed detailed research into those $P p N F-Y$ s. In addition, the identification of drought-responsive cis-elements in the promoter regions of $P p N F-Y \mathrm{~s}$ was useful to analyse and extend the drought-resistance pathway in peach. This study explored the performance of NF$Y$ s under drought stress in a notable current research area. Moreover, the current study identified two PpNF-Ys (PpNF$Y B 2$ and $P p N F-Y A 5)$ as candidate genes for drought resistance, providing a foundation for further investigating the functions of PpNF-Ys and the molecular mechanism of peach drought-resistance.

\section{Additional files}

Additional file 1: Peach NF-YS gene sequence. (DOCX $32 \mathrm{~kb}$ )

Additional file 2: Peach NF-Ys protein sequence. (DOCX $18 \mathrm{~kb}$ )

Additional file 3: Arabidopsis protein sequence. (XLSX $11 \mathrm{~kb}$ )

Additional file 4: Primer of Peach NF-Y family. (DOCX 19 kb)

\begin{abstract}
Abbreviations
$\mu \mathrm{L}$ : Microlitre; ABRE: Abscisic Acid Responsive Element; AtNF-Y: Arabidopsis NF-Y; CaNF-Y: Cicer arietinum NF-Y; CDNA: Complementary DNA; CsNFY: Citrus sinensis NF-Y; DNA: Deoxyribonucleic acid; IBS: Illustrator for Biological Sequences; Kb: Kilobase; MBS: MYB binding site involved in drought-inducibility; mRNA: Messenger RNA; Mw: Molecular weight; NFY: Nuclear Factor Y; NJ: Neighbour-joining; Pd: Prunus davidiana; Pi: Isoelectric point; PpNF-Y: Peach NF-Y; RcNF-Y: Ricinus communis NF-Y; RNA: Ribonucleic acid
\end{abstract}

\section{Acknowledgement}

We sincerely thank Bo Li (Shandong Institute of Pomology, Taian, Shandong, China) for insightful discussions.

\section{Authors' contributions}

ANZ planned and designed the research. ML, GXL, WL and XMD performed experiments, analyzed data and made conclusions based on results. ANZ and $\mathrm{ML}$ wrote the manuscript. All authors read and approved the final manuscript.

\section{Funding}

This study is supported by China Agriculture Research System (CARS-30-Z08). The funding body had no contribution in the design of the study and collection, analysis, and interpretation of data and in writing the manuscript.

\section{Availability of data and materials \\ Additional file 1. Peach NF-Ys gene sequence \\ Additional file 2. Peach NF-Ys protein sequence \\ Additional file 3. Arabidopsis protein sequence \\ Additional file 4. Primer of Peach NF-Y family}

Ethics approval and consent to participate

Not applicable.

\section{Consent for publication}

Not applicable.

\section{Competing interests}

The authors declare that they have no competing interests.

Received: 17 January 2019 Accepted: 11 July 2019

Published online: 26 July 2019

\section{References}

1. Alves PC, Hartmann DO, Nunez O, Martins I, Gomes TL, Garcia H, Galceran MT, Hampson R, Becker JD, Pereira CS. Transcriptomic and Metabolomic Profiling of Ionic Liquid Stimuli Unveils Enhanced Secondary Metabolism in Aspergillus Nidulans. BMC Genomics. 2016;17(1):284.

2. Ben S, Guasmi F, Mohamed MB, Benhaj K, Boussora F, Triki T, Kammoun NG. Identification of Internal Control Genes for Gene Expression Studies in Olive Mesocarp Tissue during Fruit Ripening. S Arf J Bo. 2018;117:11-6.

3. Berardini TZ, Reiser L, Li D, Mezheritsky Y, Muller R, Strait E, Huala E. The Arabidopsis information resource: Making and mining the "gold standard" annotated reference plant genome. Genesis. 2015;53(8):474-85.

4. Blanc $\mathrm{G}$, Wolfe $\mathrm{KH}$. Widespread paleopolyploidy in model plant species inferred from age distributions of duplicate genes. Plant Cell. 2004;16(7):1667-16785.

5. Boulard C, Thévenin J, Tranquet O, Laporte V, Lepiniec L, Dubreucq B. LEC1 (NF-YB9) directly interacts with LEC2 to control gene expression in seed. Biochim Biophys Acta - Gene Regul Mech. 2018;1861(5):443-50. 
6. Cai X, Zhang Y, Zhang C, Zhang T, Hu T, Ye J, Zhang J, Wang T, Li H, Ye Z. Genome-wide analysis of plant-specific Dof transcription factor family in tomato. J Integr Plant Biol. 2013;55(6):552-66.

7. Cao K, Zhou Z, Wang Q, Guo J, Zhao P, Zhu G, Fang W, Chen C, Wang X, Wang X, Tian Z, Wang L. Genome-Wide Association Study of 12 Agronomic Traits in Peach. Nat Commun. 2016;8(7):13246.

8. Chen M, Zhao Y, Zhuo C, Lu S, Guo Z. Overexpression of a NF-YC transcription factor from bermudagrass confers tolerance to drought and salinity in transgenic rice. Plant Biotechnol J. 2014;13(4):482-91.

9. Chu HD, Nguyen KH, Watanabe Y, Le DH, Pham TLT, Mochida K, Tran LSP. Identification, structural characterization and gene expression analysis of members of the Nuclear Factor-Y family in chickpea (Cicer arietinum L.) under dehydration and abscisic acid treatments. Int J Mol Sci. 2018;19:E3290.

10. Garcia-Albornoz M, Thankaswamy-Kosalai S, Nilsson A, Varemo L, Nookaew I, Nielsen J. BioMet Toolbox 2.0: genome-wide analysis of metabolism and omics data. Nucleic Acids Res. 2014;42(1):175-81.

11. Giovannoni JJ. Genetic regulation of fruit development and ripening. Plant Cell. 2004;16:170-80.

12. Gnesutta N, Kumimoto RW, Swain S, Chiara M, Siriwardana C, Horner DS, Holt BF, Mantovani R. CONSTANS Imparts DNA Sequence Specificity to the Histone Fold NF-YB/NF-YC Dimer. Plant Cell. 2017;29(6):1516-32.

13. Hackenberg D, Keetman U. GrimmB. Homologous NF-YC2 Subunit from Arabidopsis and Tobacco Is Activated by Photooxidative Stress and Induces Flowering. Int J Mol Sci. 2012;13(3):3458-77.

14. Hu B, Jin J, Guo A, Zhang H, Luo J, Gao G. GSDS 2.0: an upgraded gene feature visualization server. Bioinformatics. 2015;31(8):1296-7.

15. Initiative, I.P.G, Verde I, Abbott AG, Scalabrin S, Jung S, Shu S, Marroni F, Zhebentyayeva T, Dettori MT, Grimwood J, Cattonaro F, Zuccolo A, Rossini L, Jenkins J, Vendramin E, Meisel LA, Decroocq V, Sosinski B, Prochnik S, Mitros T, Policriti A, Cipriani G, Dondini L, Ficklin S, Goodstein DM, Xuan P, Fabbro CD, Aramini V, Copetti D, Gonzalez S, Horner DS, Falchi R, Lucas S, Mica E, Maldonado J, Lazzari B, Bielenberg D, Pirona R, Miculan M, Barakat A, Testolin R, Stella A, Tartarini S, Tonutti P, Arus P, Orellana A, Wells C, Main D, Vizzotto G, Silva H, Salamini F, Schmutz J, Morgante M, Rokhsar DS. The High-Quality Draft Genome of Peach (Prunus Persica) Identifies Unique Patterns of Genetic Diversity, Domestication and Genome Evolution. Nat Genet. 2013;45(5): 487-94.

16. Jung $S$, Lee $T$, Cheng $C$, Buble $K$, Zheng $P$, Yu J, Humann J, Ficklin SP, Gasic K, Scott K, Frank M, Ru S, Hough H, Evans K, Peace C, Olmstead M, DeVetter LW, McFerson J, Coe M, Wegrzyn JL, Staton ME, Abbott AG, Main D. 15 Years of GDR: New Data and Functionality in the Genome Database for Rosaceae. Nucleic Acids Res. 2018;47(1):137-45.

17. Katiyar A, Smita S, Lenka SK, Rajwanshi R, Chinnusamy V, Bansal KC. Genome-wide classification and expression analysis of MYB transcription factor families in rice and Arabidopsis. BMC Genomics. 2012;13:544.

18. Lee SC, Kim SH, Kim SR. Drought inducible OsDhn1 promoter is activated by OsDREB1A and OsDREB1D. J Plant Biol. 2013:56(2):115-21.

19. Lescot M, Dehais P, Thijs G, Marchal K, Moreau Y, Peer VY, Rouze P, Rombauts $S$. PlantCARE, a database of plant cis-acting regulatory elements and a portal to tools for in silico analysis of promoter sequences. Nucleic Acids Res. 2002;30:325-7.

20. Li S, Li K, Ju Z, Cao D, Fu D, Zhu H, Zhu B, Luo Y. Genome-wide analysis of tomato NF-Y factors and their role in fruit ripening. BMC Genomics. 2016;17:36

21. Link J, Pachaly J. Intranarcotic infusion therapy: A computer interpretation using the program package SPSS (Statistical package for the social sciences). Infusionsther Klin Ernahr. 1975;2(4):255-9.

22. Liu W, Xie Y, Ma J, Luo X, Nie P, Zuo Z, Lahrmann U, Zhao Q, Zheng Y, Zhao $Y, X u e Y$, Ren J. BS: an illustrator for the presentation and visualization of biological sequences. Bioinformatics. 2015;31(20):3359-61.

23. Mantovani R. The molecular biology of the CCAAT-binding factor NF-Y. Gene. 1999;239(1):15-27.

24. Marchler-Bauer A, Zheng C, Chitsaz F, Derbyshire MK, Geer LY, Geer RC, Gonzales NR, Gwadz M, Hurwitz DI, Lanczycki CJ, Lu F, Lu S, Marchler GH, Song JS, Thanki N, Yamashita RA, Zhang D, Bryant SH. CDD: Conserved domains and protein three-dimensional structure. Nucleic Acids Res. 2012; 41(1):348-52.

25. Myers ZA, Holt BF. NUCLEAR FACTOR-Y: Still Complex after All These Years? Curr Opin Plant Biol. 2018:45:96-102.
26. Nakashima K, Jan A, Todaka D, Maruyama K, Goto S, Shinozaki K, Yamaguchi-Shinozaki K. Comparative functional analysis of six droughtresponsive promoters in transgenic rice. Planta. 2014;239(1):47-60.

27. Nardone V, Chaves-Sanjuan A, Nardini M. Structural determinants for NF-Y/ DNA interaction at the CCAAT box. Biochim Biophys Acta - Gene Regul Mech. 2017:1860(5):571-80.

28. Nelson DE, Repetti PP, Adams TR, Creelman RA, Wu J, Warner DC, Anstrom DC, Bensen RJ, Castiglioni PP, Donnarummo MG, Hinchey BS, Kumimoto RW, Maszle DR, Canales RD, Krolikowski KA, Dotson SB, Gutterson N, Ratcliffe OJ, Heard JE. Plant nuclear factor Y (NF-Y) B subunits confer drought tolerance and lead to improved corn yields on water-limited acres. Proc Natl Acad Sci U S A. 2007;104(42):16450-5.

29. Ni Z, Zheng H, Jiang Q, Zhang H. GmNFYA3, a target gene of miR169, is a positive regulator of plant tolerance to drought stress. Plant Mol Biol. 2013;82(1-2):113-29.

30. Nicholas KB, Nicholas HB, Deerfield DW, Nicholas HBJ, Nicholas K, Nicholas HJ, Nicholas KR, Nicholas HBJ, Nicholas A, Deerfield DW, Nicholas H, Gauch H. GeneDoc: a tool for editing and annotating multiple sequence alignments. Embnet.news. 1997.

31. Nordberg H, Cantor M, Dusheyko S, Hua S, Poliakov A, Shabalov I, Smirnova T, Grigoriev IV, Dubchak I. The genome portal of the Department of Energy Joint Genome Institute: 2014 updates. Nucleic Acids Res. 2014;42:26-31.

32. Olias R, Eljakaoui Z, Li J, De M, Alvarez P, Marin-Manzano MC, Pardo JM, Belver A. The plasma membrane $\mathrm{Na}+/ \mathrm{H}+$ antiporter SOS1 is essential for salt tolerance in tomato and affects the partitioning of $\mathrm{Na}+$ between plant organs. Plant Cell Environ. 2009;32(7):904-16.

33. Pereira SLS, Martins CPS, Sousa AO, Camillo LR, Araujo CP, Alcantara GM, Camargo DS, Cidade LC, de Almeida AF, Costa MGC. Genome-wide characterization and expression analysis of citrus NUCLEAR FACTOR-Y (NF-Y) transcription factors identified a novel NF-YA gene involved in drought-stress response and tolerance. PLoS One. 2018;13(6):e0199187.

34. Rozas J, Ferrer-Mata A, Sanchez-DelBarrio JC, Guirao-Rico S, Librado P, Ramos-Onsins SE, Sanchez-Gracia A. DnaSP 6: DNA Sequence Polymorphism Analysis of Large Datasets. Mol Biol Evol. 2017;34(12):3299-302.

35. Saitou N, Nei M. The Neighbor-Joining Method-a New Method for Reconstructing Phylogenetic Trees. Mol Biol Evol. 1987;4(4):406-25.

36. Shi J, Zhang L, An H, Wu C, Guo X. GhMPK16, a novel stress-responsive group DMAPK gene from cotton, is involved in disease resistance and drought sensitivity. BMC Mol Biol. 2011;12(1):22.

37. Siefers N, Dang K, Kumimoto R, Bynum W, Tayrose G, Holt B. TissueSpecific Expression Patterns of Arabidopsis NF-Y Transcription Factors Suggest Potential for Extensive Combinatorial Complexity. Plant Physiol. 2009;149(2):625-41.

38. Song XM, Liu TK, Duan WK, Ma QH, Ren J, Wang Z, Li Y, Hou XL. Genome-Wide Analysis of the GRAS Gene Family in Chinese Cabbage (Brassica Rapa Ssp. Pekinensis). Genomics. 2014;103(1):135-46.

39. Swain S, Myers Z, Chamindika S, Holt B. The Multifaceted Roles of NUCLEAR FACTOR-Y in Arabidopsis Thaliana Development and Stress Responses. Biochim Biophys Acta - Gene Regul Mech. 2016;1860(5):636-44.

40. Tamura K, Peterson D, Peterson N, Stecher G, Nei M, Kumar S. MEGA5: Molecular Evolutionary Genetic Analysis using Maximum Likelihood, Evolutionary Distance, and Maximum Parsimony Methods. Mol Biol Evol. 2011;28(10):2731-9.

41. Thon M, Al Abdallah Q, Hortschansky P, Scharf DH, Eisendle M, Haas H, Brakhage AA. The CCAAT-binding complex coordinates the oxidative stress response in eukaryotes. Nucleic Acids Res. 2010;38(4):1098-113.

42. Timothy B, Elkan C. Fitting a mixture model by expectation maximization to discover motifs in bipolymers. Proc Int Conf Intell Syst Mol Biol. 1994;2:28-36.

43. Tong Z, Gao Z, Wang F, Zhou J, Zhang Z. Selection of reliable reference genes for gene expression studies in peach using real-time PCR. BMC Mol Biol. 2009;10:71.

44. Wang L, Zhu J, Li X, Wang S, Wu J. Salt and Drought Stress and ABA Responses Related to BZIP Genes from V. Radiata and V. Angularis. Gene. 2018a;651(20):152-60.

45. Wang Y, Xu W, Chen Z, Han B, Haque ME, Liu A. Gene structure, expression pattern and interaction of Nuclear Factor-Y family in castor bean (Ricinus communis). Planta. 2018b;247:559-72.

46. Yan H, Wu F, Jiang G, Xiao L, Li Z, Duan X, Jiang Y. Genome-wide identifcation, characterization and expression analysis of NFY gene 
family in relation to fruit ripening in banana. Postharvest Biol Technol. 2019;151:98-110.

47. Yang W, Lu Z, Xiong Y, Yao J. Genome-wide identification and coexpression network analysis of the OsNF-Y gene family in rice. Crop J. 2017;5(1):21-31.

48. Zambelli F, Pavesi G. Genome wide features, distribution and correlations of NF-Y binding sites. Biochim. Biophys. Acta - Gene Regul. Mech. 2017;1860(5):581-9.

49. Zanetti ME, Ripodas C, Niebel A. Plant NF-Y transcription factors: Key players in plant-microbe interactions, root development and adaptation to stress. Biochim Biophys Acta - Gene Regul Mech. 2017;1860(5):645-54.

50. Zhang H, Gao S, Lercher MJ, Hu S, Chen WH. EvolView, an online tool for visualizing, annotating and managing phylogenetic trees. Nucleic Acids Res. 2012:40(1):569-72.

51. Zhang H, Kang H, Su C, Qi Y, Liu X, Pu J. Genome-Wide Identification and Expression Analysis of the NAC Transcription Factor Family in Cassava. PLoS One. 2015a;10(6):e0136993.

52. Zhang Z, Li X, Yu R, Han M, Wu Z. Isolation, structural analysis, and expression characteristics of the maize TIFY gene family. Mol Gen Genomics. 2015b;290(5):1849-58.

\section{Publisher's Note}

Springer Nature remains neutral with regard to jurisdictional claims in published maps and institutional affiliations.

Ready to submit your research? Choose BMC and benefit from:

- fast, convenient online submission

- thorough peer review by experienced researchers in your field

- rapid publication on acceptance

- support for research data, including large and complex data types

- gold Open Access which fosters wider collaboration and increased citations

- maximum visibility for your research: over $100 \mathrm{M}$ website views per year

At BMC, research is always in progress.

Learn more biomedcentral.com/submissions 